\title{
Effects of Dexmedetomidine on Perioperative Serum Potassium and Postoperative Rehabilitation of Patients Undergoing Radical Resection of Gastrointestinal Malignancy
}

\section{Shunping Tian}

the Affiliated Hospital of Yangzhou University, Yangzhou University

\section{Dongsheng Zhang}

the Affiliated Hospital of Yangzhou University, Yangzhou University

\section{Ying Wang}

Dalian Medical University

\section{Leyang Yu}

Dalian Medical University

\section{Yanlong Yu}

the Affiliated Hospital of Yangzhou University, Yangzhou University

\section{Ning Li}

the Affiliated Hospital of Yangzhou University, Yangzhou University

Shiting Yan

Dalian Medical University

\section{Wei Zhou}

the Affiliated Hospital of Yangzhou University, Yangzhou University

Zhuan Zhang ( $\nabla$ zhangzhuancg@163.com )

the Affiliated Hospital of Yangzhou University, Yangzhou University

\section{Research Article}

Keywords: Dexmedetomidine, Electrolyte, Malignancy, Gastrointestinal tract, Rehabilitation

Posted Date: December 29th, 2021

DOI: https://doi.org/10.21203/rs.3.rs-1169113/v1

License: (a) (i) This work is licensed under a Creative Commons Attribution 4.0 International License. Read Full License 


\section{Abstract}

Background: To investigate the effects of dexmedetomidine on perioperative potassium and postoperative rehabilitation.

Methods: Totally 124 patients scheduled for elective radical resection of gastrointestinal malignant tumor under general anesthesia were included. and randomly assigned to four groups $(n=31)$ : groups $D_{1}, D_{2}$ and $D_{3}$ received dexmedetomidine loading dose 1,1 and $0.5 \mu \mathrm{g} / \mathrm{kg}$ and maintenance dose $0.25,0.5$ and $0.5 \mu \mathrm{g} / \mathrm{kg} / \mathrm{h}$, respectively, group $\mathrm{C}$ received normal saline $50 \mathrm{ml} / \mathrm{h}$ for $10 \mathrm{~min}$ and maintenance dose $10 \mathrm{ml} / \mathrm{h}$. Serum potassium and lactate changes were recorded at $5 \mathrm{~min}$ after arteriovenous puncture $\left(\mathrm{T}_{1}\right), 1 \mathrm{~h}$ after surgery beginning $\left(T_{6}\right)$, surgery ending $\left(T_{7}\right)$ and $1 \mathrm{~h}$ into the post-anesthesia care unit (PACU) $\left(T_{10}\right)$. Serum potassium was examined at $48 \mathrm{~h}$ after surgery $\left(\mathrm{T}_{11}\right)$. Perioperative rehabilitation indicators were recorded.

Results: Compared with basal values, serum potassium concentration in groups $C, D_{1}$ and $D_{2}$ increased significantly at $\mathrm{T}_{11}\left(P=0.003,0.002\right.$, and $\nabla 0.001$, respectively) and at $\mathrm{T}_{7}(P=0.008), \mathrm{T}_{10}(P=0.015)$ and $\mathrm{T}_{11}$ $(P \otimes 0.001)$ in group $\mathrm{D}_{3}$. Serum potassium at $\mathrm{T}_{11}$ in group $\mathrm{D}_{2}$ was significantly lower than group $\mathrm{C}(P=0.032)$. Serum potassium at $T_{7}$ in group $D_{3}$ was significantly higher than group $D_{2}(P=0.036)$. There were no significant differences in perioperative rehabilitation indicators in all groups.

Conclusions: Dexmedetomidine did not decrease perioperative potassium significantly in patients undergoing radical resection of gastrointestinal malignancy, while its loading dose $0.5 \mu \mathrm{g} / \mathrm{kg}$ and maintenance dose 0.5 $\mu \mathrm{g} / \mathrm{kg} / \mathrm{h}$ can elevate potassium slightly with no adverse effect on perioperative rehabilitation.

Trial registration: This study was retrospectively registered on the clinicaltrials.gov website (registration number: NCT04771637).

\section{Introduction}

Dexmedetomidine is a highly selective $\mathrm{a}_{2}$-adrenergic receptor agonist, which is often used in clinical anesthesia and intensive care unit due to its sedative, analgesic and sympathetic suppressing effects[1]. For radical gastrointestinal malignancy surgery with long operation time and heavy trauma, dexmedetomidine is often used during general anesthesia to blunt strong stress response. Patients with gastrointestinal malignant tumors often harbor several risk factors such as advanced age, poor nutritional state owing to appetite loss and combined underlying diseases[2], which, with prolonged preoperative fasting, are prone to inducing electrolyte disorders. While prolonged use of dexmedetomidine is associated with a risk of prolonging QT interval and causing the incidence of tip torsion ventricular tachycardia[3]. Perioperative hypokalemia might occur during gastrointestinal surgery and affect myocardial repolarization to increase the risk of tip torsion ventricular tachycardia[4]. When severe, potassium disorders can lead to life-threatening cardiac conduction disturbances and neuromuscular dysfunction[5]. It is still not clear whether prolonged use of dexmedetomidine during gastrointestinal malignancy radical resection could induce or exacerbate hypokalemia or not.

The primary aim of this prospective, randomized, doubled-blind study is to observe the effects of prolonged use of dexmedetomidine on electrolytes concentration and postoperative rehabilitation. The second aim is to 
explore the optimal dose of dexmedetomidine with the least effect on serum potassium concentration in patients undergoing radical resection of gastrointestinal tumors.

\section{Materials And Methods}

This study was approved by the Ethics Committee of the Affiliated Hospital of Yangzhou University (ethics number: 2017-YKL11-01) and registered on the clinicaltrials.gov website (registration number: NCT04771637). After obtaining informed written consent for participation in the study, patients aged 55-75 years of age, body mass index (BMI) $18.5-25 \mathrm{~kg} / \mathrm{m}^{2}$ and American Society of Anesthesiologists status I-III of both genders underwent general anesthesia for elective radical resection of gastrointestinal malignancy with an estimated operation time longer than 3 hours were enrolled in this study between January 2017 and October 2020. Patients were not admitted to the study if any of the following criteria were present: severe sinus bradycardia (heart rate $<50$ beats/min), pacemaker implantation, atrioventricular block, severe circulatory disturbance during operation, malignant arrhythmia, impairment of liver or kidney, preoperative severe internal environmental disorders such as hypokalemia (serum potassium level $<3.0 \mathrm{mmol} / \mathrm{L}$ ), hyperkalemia (serum potassium level $>5.5 \mathrm{mmol} / \mathrm{L}$ ), hyperlactatemia (blood lactate concentration $>2.0 \mathrm{mmol} / \mathrm{L}$ ), intraoperative blood transfusion and postoperative need to be transferred to intensive care unit for further treatment.

\subsection{Randomization and blinding}

Eligible patients were randomly assigned to one of four groups according to a computer generated table of random numbers: groups $D_{1}, D_{2}$ and $D_{3}$ received dexmedetomidine loading dose 1,1 and $0.5 \mu \mathrm{g} / \mathrm{kg}$, which was infused intravenously for $10 \mathrm{~min}$, and maintenance dose $0.25,0.5$ and $0.5 \mu \mathrm{g} / \mathrm{kg} / \mathrm{h}$, respectively, group C received normal saline $50 \mathrm{ml} / \mathrm{h}$ for $10 \mathrm{~min}$ and maintenance dose $10 \mathrm{ml} / \mathrm{h}$. Dexmedetomidine and normal saline maintenance doses in the four groups were continued until approximately $30 \mathrm{~min}$ before surgery completion. The sealed opaque envelope technique was used for group allocation. A researcher not involved in the study implementation opened the envelopes. Patients, surgeons, and the outcome assessor were blinded to the technique used.

\subsection{Anesthesia}

None of the patients received preoperative medication. Routine monitoring including electrocardiograph (ECG), pulse oxygen saturation $\left(\mathrm{SpO}_{2}\right)$ and noninvasive blood pressure (NBP) were performed after patients entering the operating room. Narcotrend monitoring (MT MonitorTechnik GmbH\&Co.KG, Germany) was used to monitor the depth of anesthesia. Ultrasound-guided right internal jugular vein puncture was performed under local anesthesia, and a double-lumen catheter was inserted to a depth of $12-13 \mathrm{~cm}$. After that, a combination of $0.9 \%$ sodium chloride solution and $6 \%$ hydroxyethyl starch solution $130 / 0.4$ at a ratio of $1: 2$ was infused at a rate of $6-7 \mathrm{~mL} / \mathrm{kg} / \mathrm{h}$. Patients with a normal result on the modified Allen's test were catheterized with radial artery puncture under local anesthesia to monitor invasive arterial pressure (IAP). Vital signs [mean blood pressure (MAP) and heart rate (HR)] were recorded 5 min after arteriovenous puncture. Groups $D_{1}, D_{2}$, and $D_{3}$ were given an intravenous injection of dexmedetomidine (18092931, 19012631, 19081331, Yangzijiang Pharmaceutical Co., Ltd.) with the loading dose for 10 min before general anesthesia induction and the maintenance dose until approximately $30 \mathrm{~min}$ before the end of surgery. Group $\mathrm{C}$ was given intravenous normal saline at a rate of $50 \mathrm{~mL} / \mathrm{h}$ for $10 \mathrm{~min}$, followed by continuous infusion at a rate of $10 \mathrm{~mL} / \mathrm{h}$ until about $30 \mathrm{~min}$ 
before the end of surgery. Dexmedetomidine or normal saline was prepared by the study personnel who did not participate in data collection, and the syringe pumped through the fourth channel of a syringe pump (CP-2100, Beijing Silugao Medical Technology Co., Ltd., China) was covered with a piece of opaque sheet.

General anesthesia induction was carried out with midazolam $0.05 \mathrm{mg} / \mathrm{kg}$, sufentanil $4 \mu \mathrm{g} / \mathrm{kg}$ and propofol 1.5 $\mathrm{mg} / \mathrm{kg}$ in all groups. Endotracheal intubation and mechanical ventilation were performed after sufficient muscle relaxation with cisatracurium $0.2 \mathrm{mg} / \mathrm{kg}$. Mechanical ventilation was maintained with inhaled oxygen concentration $60 \%$, tidal volume $6-8 \mathrm{~mL} / \mathrm{kg}$, ventilation frequency $12-14$ times $/ \mathrm{min}$, and inspiration/expiration ratio 1:1.5 to maintain $\mathrm{P}_{\mathrm{ET}} \mathrm{CO}_{2}$ at $35-45 \mathrm{mmHg}$. Temperature was maintained at $36^{\circ} \mathrm{C}-37^{\circ} \mathrm{C}$. General anesthesia maintenance was implemented with intravenous propofol and remifentanil and inhaled sevoflurane $1 \mathrm{MAC}$ in all groups. Muscle relaxation was maintained with cisatracurium infusion at $0.15 \mathrm{mg} \cdot \mathrm{kg}^{-1} \cdot \mathrm{h}^{-1}$. The doses of propofol and remifentanil were adjusted according to the Narcotrend monitoring, the stage of which was maintained between D2 to E1 (sedation index 20-46) throughout surgery in all groups. Train of four (TOF) monitoring was used to monitor the muscle relaxation during the surgery. When the changes of IAP or HR were $>20 \%$ of basic levels, prepared vasoactive drugs including norepinephrine, ephedrine or atropine was used. Sevoflurane and cisatracurium were stopped at about 30 minutes before surgery ending, and propofol and remifentanil were continued until the end of surgery. Residual neuromuscular blockade was antagonized at TOF ratio more than 0.75 with intravenous neostigmine $0.04-0.07 \mathrm{mg} / \mathrm{kg}$ and atropine $0.01-0.02 \mathrm{mg} / \mathrm{kg}$. Patients were extubated when achieved extubation conditions and transferred to PACU thereafter.

\subsection{Outcome variables}

MAP and HR were recorded at 5 min after artery puncture $\left(T_{1}\right)$, dexmedetomidine loading dose finish $\left(T_{2}\right)$, surgery beginning $\left(T_{3}\right), 5 \mathrm{~min}\left(T_{4}\right), 10 \mathrm{~min}\left(T_{5}\right)$ and $1 \mathrm{~h}\left(T_{6}\right)$ after surgery beginning, surgery ending $\left(T_{7}\right)$, extubation $\left(T_{8}\right), 5$ min after extubation $\left(T_{9}\right)$ and 1 hour after into PACU $\left(T_{10}\right)$. Serum potassium and lactic acid were recorded at $T_{1}, T_{6}, T_{7}$ and $T_{10}$. Serum potassium was also examined at $24 \mathrm{~h}$ postoperatively $\left(T_{11}\right)$. Liver and kidney function, intraperitoneal drainage volume at $24 \mathrm{~h}$ postoperatively, length of hospital stay and postoperative complications such as pulmonary infection were also recorded.

\subsection{Statistical analysis}

The necessary sample size was calculated using PASS 15.0 (NCSS LIc., USA). The sample size calculation was based on the primary endpoint of this study, serum potassium concentration. Based on our pilot study, serum potassium concentrations in patients treated with dexmedetomidine infusion were found to have an average increase of $0.24 \mathrm{mmol} / \mathrm{L}$ with a standard deviation of $0.36 \mathrm{mmol} / \mathrm{L}$. Consequently, enrollment of 25 patients in each group would obtain a power of $80 \%(\beta=0.2)$ at a significant level of $0.05(\alpha=0.05$, two-tailed). Considering study withdrawals or protocol violation, recruitment of 30 in each group was determined.

Statistical analysis was done using SPSS 25.0 (SPSS Inc., USA). Parametric data were expressed in means \pm standard deviation (SD), and Student's $t$ test was used for analyses. Nominal variables were compared using chi-square test. Changes in mean blood pressure and heart rate among groups were analyzed by repeated measures ANOVA. $P<0.05$ was considered significant.

\section{Results}


A total of 140 patients were assessed for eligibility, and 124 subjects were enrolled in the study. The remaining 16 patients were excluded because either they did not meet the inclusion criteria $(n=10)$ or they refused to participate $(n=6)$. Thirty-one patients were allocated to each group. Due to protocol violation, 1 patient were excluded from group $D_{2}$. Therefore, 31 patients in group $D_{1}, 30$ patients in group $D_{2}, 31$ patients in group $D_{3}$, and 31 patients in group $\mathrm{C}$ were analyzed (Figure 1).

There were no significant differences of the demographics among the four groups (Table 1). Compared with group $C$, urine volume increased significantly in groups $D_{2}$ and $D_{3}(P=0.004$ and 0.039 , respectively), while other intraoperative data were similar among the four groups (Table 2).

\subsection{Potassium and lactate}

Compared with the basic serum potassium values, the concentrations at $T_{11}$ increased significantly in groups $\mathrm{D}_{1}, \mathrm{D}_{2}$ and $\mathrm{C}\left(P=0.003,0.002\right.$, and $\nabla 0.001$, respectively), while increased significantly at $\mathrm{T}_{7}(P=0.008), \mathrm{T}_{10}$ $(P=0.015)$ and $\mathrm{T}_{11}(P \otimes 0.001)$ in group $\mathrm{D}_{3}$. Compared with group $\mathrm{C}$, the serum potassium concentration in group $\mathrm{D}_{2}$ decreased significantly at $\mathrm{T}_{11}(P=0.032)$. Compared with group $\mathrm{D}_{2}$, the serum potassium concentration in group $D_{3}$ increased significantly at $T_{7}(P=0.036)$.

Compared with the basic serum lactate values, the concentrations decreased significantly at $T_{6}$ in groups $D_{2}$ and $D_{3}\left(P=0.002\right.$ and 0.003 , respectively). Compared with group $D_{2}$, the serum lactate concentrations in group $\mathrm{D}_{3}$ decreased significantly at $\mathrm{T}_{10}(P=0.033)$ (Table 3$)$.

\subsection{Hemodynamic results}

Repeated-measures analysis of variance revealed significant variation in HR and MAP over time within each group (Table 4, 5).

Compared with $\mathrm{T}_{1}, \mathrm{HR}$ decreased significantly at $\mathrm{T}_{2}(P=0.002), \mathrm{T}_{3}(P=0.001), \mathrm{T}_{4}(P \llbracket 0.001), \mathrm{T}_{5}(P \llbracket 0.001), \mathrm{T}_{6}$ $(P=0.003)$ and $\mathrm{T}_{7}(P=0.033)$ in group $\mathrm{D}_{1}$, at $\mathrm{T}_{3}(P \otimes 0.001), \mathrm{T}_{4}(P \otimes 0.001), \mathrm{T}_{5}(P \otimes 0.001), \mathrm{T}_{6}(P \otimes 0.001), \mathrm{T}_{7}(P \otimes 0.001)$, $\mathrm{T}_{8}(P=0.030), \mathrm{T}_{9}(P=0.037)$ and $\mathrm{T}_{10}(P \otimes 0.001)$ in group $\mathrm{D}_{2}$, at $\mathrm{T}_{2}(P=0.025), \mathrm{T}_{3}(P=0.001), \mathrm{T}_{4}(P \otimes 0.001), \mathrm{T}_{5}$ $(P \otimes 0.001), \mathrm{T}_{6}(P \otimes 0.001), \mathrm{T}_{7}(P \otimes 0.001), \mathrm{T}_{8}(P=0.024), \mathrm{T}_{9}(P=0.022)$ and $\mathrm{T}_{10}(P \otimes 0.001)$ in group $\mathrm{D}_{3}$, and at $\mathrm{T}_{4}$ $(P=0.023)$ in group $C$. Compared with group $C, H R$ decreased significantly in group $D_{2}$ at $T_{5}(P=0.010), T_{7}$ $(P=0.017), \mathrm{T}_{8}(P=0.005), \mathrm{T}_{9}(P=0.030)$ and $\mathrm{T}_{10}(P=0.011)$ and in group $\mathrm{D}_{3}$ at $\mathrm{T}_{5}(P=0.042), \mathrm{T}_{7}(P=0.002), \mathrm{T}_{8}$ $(P=0.007), \mathrm{T}_{9}(P=0.034)$ and $\mathrm{T}_{10}(P=0.019)$.

Compared with $\mathrm{T}_{1}, \mathrm{MAP}$ at $\mathrm{T}_{4}(P=0.002), \mathrm{T}_{5}(P=0.007), \mathrm{T}_{6}(P \otimes 0.001), \mathrm{T}_{7}(P \otimes 0.001), \mathrm{T}_{8}(P=0.047)$ and $\mathrm{T}_{9}$ $(P=0.048)$ decreased significantly in group $\mathrm{D}_{1}$, at $\mathrm{T}_{10}(P=0.029)$ in group $\mathrm{D}_{2}$, and at $\mathrm{T}_{6}(P=0.006)$ and $\mathrm{T}_{10}$ $(P=0.002)$ in group $\mathrm{D}_{3}$, while decreased significantly at $\mathrm{T}_{3}(P=0.007)$ and $\mathrm{T}_{6}(P=0.002)$ and increased significantly at $\mathrm{T}_{8}(P=0.015)$ in group $C$. Compared with group $C$, MAP increased significantly at $\mathrm{T}_{2}$ $(P=0.040)$ and decreased significantly at $\mathrm{T}_{5}(P=0.045), \mathrm{T}_{7}(P=0.018), \mathrm{T}_{8}(P=0.001)$ and $\mathrm{T}_{9}(P=0.030)$ in group $\mathrm{D}_{1}$, decreased significantly at $\mathrm{T}_{8}(P=0.002), \mathrm{T}_{9}(P=0.049)$ and $\mathrm{T}_{10}(P=0.037)$ in group $\mathrm{D}_{2}$, and at $\mathrm{T}_{8}(P=0.033)$ and $\mathrm{T}_{9}(P=0.013)$ in group $\mathrm{D}_{3}$. 


\subsection{Perioperative drug use}

Significantly, less dosage of propofol was required $(P=0.011)$ in group $D_{2}$ as compared to Group $C$. Again, dose of remifentanil was significantly lower in groups $D_{1}, D_{2}$ and $D_{3}(P=0.039,0.004$ and 0.042 , respectively) than group $C$. More patients in group $C$ received ephedrine to treat intraoperative hypotension $(P=0.048)$ than the other groups.

\subsection{Postoperative recovery indicators}

The activity of plasma alanine aminotransferase (ALT) was significantly higher at $T_{11}$ than $T_{1}$ in all groups $\left(P=0.030, \varangle 0.001,0.005\right.$ and 0.011 in groups $D_{1}, D_{2}, D_{3}$ and $C$, respectively), and plasma aspartate aminotransferase (AST) activities increased significantly at $\mathrm{T}_{11}$ than $\mathrm{T}_{1}$ in groups $\mathrm{D}_{2}, \mathrm{D}_{3}$ and $\mathrm{C}(P=0.037,0.034$ and 0.031 , respectively). There was no significant difference in serum creatinine. Additionally, patients in group $\mathrm{D}_{1}$ had significantly higher blood urea nitrogen at $\mathrm{T}_{11}$ than $\mathrm{T}_{1}(P=0.014)$ (Table 6). There were no significant differences in length of hospital stay, intraperitoneal drainage volume and pulmonary infection incidence at $24 \mathrm{~h}$ postoperatively among the four groups.

\section{Discussion}

The results of this prospective double-blind randomized study suggest that dexmedetomidine loading dose 0.5 $\mu \mathrm{g} / \mathrm{kg}$ and maintenance dose $0.5 \mu \mathrm{g} / \mathrm{kg} / \mathrm{h}$ could increase serum potassium concentration slightly during perioperative period.

The incidence of hypokalemia resulted from dexmedetomidine when administered for long-term sedation in the intensive care unit setting was reported to be more than $2 \%$ in adult[6] and more than $9 \%$ in newborns[7], the mechanism of which is still uncertain. Görges et al.[4] reported that potassium levels were depressed relative to baseline at $15 \mathrm{~min}$ and $30 \mathrm{~min}$ after intravenous injection of dexmedetomidine as a bolus administered over 60 seconds after general anesthesia induction. However, different from the results of the previous studies, dexmedetomidine did not produce a decreased effect on potassium levels in our study. We found an increase in serum potassium concentration at surgery ending, $5 \mathrm{~min}$ after extubation and $24 \mathrm{~h}$ postoperatively. when dexmedetomidine loading dose $0.5 \mu \mathrm{g} / \mathrm{kg}$ and maintenance dose $0.5 \mu \mathrm{g} / \mathrm{kg} / \mathrm{h}$ was used. The mechanism by which dexmedetomidine causes a mild increase in serum potassium is still unclear.

Catecholamine-induced vasoconstriction results in peripheral tissue ischemia, which causes anaerobic metabolism and lactate production in peripheral tissue[8]. Dexmedetomidine can reduce the sympathetic nervous system activity and is useful in diminishing catecholamine release through its anti-sympatholytic properties[9]. Therefore, dexmedetomidine may alleviate excessive catecholamine-induced lactate overproduction[10,11]. A previous study reported the impact of dexmedetomidine on tourniquet-induced systemic effects in total knee arthroplasty, in which lactic acid concentration was significantly lower in the dexmedetomidine group compared with the control group at $10 \mathrm{~min}$ after tourniquet release. The authors concluded that the reduced serum lactate level might be related to the reduction of tourniquet-induced pain and hemodynamic fluctuations, as well as the regulation of metabolism and coagulation induced from dexmedetomidine. Another study suggested that dexmedetomidine may ameliorate liver damage and decrease lactate level[12]. In the current study, serum lactate level was significantly lower in groups $D_{2}$ and $D_{3}$ at $1 \mathrm{~h}$ after 
surgery beginning compared with the basic value, suggesting that dexmedetomidine loading dose $0.5 \mu \mathrm{g} / \mathrm{kg}$ or $1 \mu \mathrm{g} / \mathrm{kg}$ and maintenance dose $0.5 \mu \mathrm{g} / \mathrm{kg} / \mathrm{h}$ might exert certain improvement effects on microcirculatory perfusion.

A transient increase in blood pressure in a short period of time after dexmedetomidine use may be associated with its direct activation on alpha ${ }_{2}$-adrenergic receptors on vascular smooth muscle cells and leading to vasoconstriction, after which blood pressure drops with dexmedetomidine acting on the locus ceruleus, inhibiting the sympathetic nervous system excitation and also increasing the vagal excitability[13, 14]. In the current study, it is noteworthy that MAP was significantly higher in group $D_{1}$ after dexmedetomidine loading dose finish compared to the control group, whereas there were no significant changes in MAP in group $D_{2}$ at this timepoint, which may be related to the higher basic MAP in the group $D_{1}$ than group $D_{2}$. Dexmedetomidine loading dose administration caused further vasoconstriction, resulting in an increase of MAP. MAP and HR in dexmedetomidine groups were significantly lower than those in the control group at several time points during operation, at extubation and at 1 hour after into PACU, which might be related to the inhibitory effect of dexmedetomidine on sympathetic nerve.

The antinociceptive effects of dexmedetomidine occurs at dorsal root neuron level, where it blocks the release of substance $P$ in the nociceptive pathway and through action on inhibitory $G$ protein $[15,16]$. We found that remifentanil dosage in each dexmedetomidine group was significantly lower than that in the control group, which might be related to its analgesic effect.

Dexmedetomidine inhibits arginine vasopressin release from the isolated hypothalamic paraventricular nucleus and paraventricular magnocellular neurons and thus producing a diuretic effect[17-19]. As reported in the current study, the urine volume in groups $D_{2}$ and $D_{3}$ was significantly higher than that in group $C$. While there were no significant changes in the urine volume in group $D_{1}$, which indicating that lower dose of dexmedetomidine might have no obvious diuretic effect. Though the blood urea nitrogen $24 \mathrm{~h}$ postoperatively in group $D_{1}$ was significantly higher than that before surgery, it was still in normal range, and the renal function in the other groups did not change significantly. Previous studies by Jiang et al.[20] have confirmed that dexmedetomidine has a protective effect on the liver. The activity of ALT and AST increased at 24 $\mathrm{h}$ postoperatively, which might be related to the decrease of the activity of digestive enzymes in combination with the decrease of nutrient digestibility due to the changes on the intestinal environment. While the ALT and AST levels were all in normal ranges. Additionally, there were no significant differences in length of hospital stay, intraperitoneal drainage volume and pulmonary infection incidence $24 \mathrm{~h}$ postoperatively among the four groups, which may be related to the modes of operation, the antibiotic treatment and standard procedure of the hospital.

There are still some shortcomings in this study. First, only patients undergoing radical resection of gastrointestinal malignant tumors were included in this study, whether the results are applicable to patients with other types of surgery still needs further study. Second, the three dosages of the dexmedetomidine were selected based on our previous studies reported in the literature. The optimal intra-operative dose of dexmedetomidine remain to be determined, and a further study is needed. Third, perioperative infusion of $0.9 \%$ sodium chloride solution may have a certain impact on electrolyte determination. Finally, randomized controlled trials of large samples and multicenter studies are needed to provide reliable evidence for the use of dexmedetomidine. 
In summary, dexmedetomidine loading dose $0.5 \mu \mathrm{g} / \mathrm{kg}$ and maintenance dose $0.5 \mu \mathrm{g} / \mathrm{kg} / \mathrm{h}$ can elevate serum potassium concentration slightly, reduce lactic acid and remifentanil use significantly, relieve perioperative hemodynamic fluctuations, and have no adverse effect on postoperative rehabilitation in patients undergoing radical resection of gastrointestinal malignancy.

\section{Abbreviations}

Electrocardiograph (ECG), Pulse oxygen saturation $\left(\mathrm{SpO}_{2}\right)$, Noninvasive blood pressure (NBP), Mean blood pressure (MAP), Heart rate (HR), Invasive arterial pressure (IAP), Body mass index (BMI), Post-anesthesia care unit (PACU), Train of four (TOF), Alanine aminotransferase (ALT): Aspartate aminotransferase (AST), Standard deviation (SD).

\section{Declarations}

Ethics approval and consent to participate: This study was approved by the Ethics Committee of the Affiliated Hospital of Yangzhou University (ethics number: 2017-YKL11-01) and registered on the clinicaltrials.gov website (registration number: NCT04771637)

Consent for publication: Not applicable.

Funding: This work was supported by the Medical Key Talents Training Project of the Yangzhou City Health and Family Planning Commission (ZDRC201815) and the Grants for Yangzhou Natural Science Foundation (YZ2017122).

Competing interests: The authors certify that there was no conflict of interest with any financial organization regarding the material discussed in the manuscript.

Availability of data and materials: The datasets used and analysed during the current study are available from the corresponding author on reasonable request.

Authors' Contributions: Zhang Zhuan designed the study, drafted and wrote the manuscript. Shunping Tian, Dongsheng Zhang, Ying Wang and Leyang Yu implemented the trial and contributed samples collection. Shinting Yan and Wei Zhou prepared drugs. Ning Li and Yanlong Yu collected the data and did statistical analysis. All authors reviewed the manuscript.

Acknowledgments: We sincerely thank Dr Zhengbing Wang, Dr. Jianming Ma, Dr Wei Xia, Dr. Ming Zhou and Dr. Hailiang Liang for their excellence in performing surgical techniques as well as all the nursing staff for their patience and enthusiastic support. We have obtained the permission to acknowledge from all those mentioned in the Acknowledgements section.

\section{References}

1. Dong Q, Li C, Xiao F, Xie Y: Efficacy and safety of dexmedetomidine in patients receiving mechanical ventilation: Evidence from randomized controlled trials. Pharmacol Res Perspect 2020, 8:e00658. 
2. Uzuka T, Takahashi H, Nakasu Y, Okuda T, Mitsuya K, Hayashi N, Hirose T, Kurai H: Surgical Site Infection after Malignant Brain Tumor Resection: A Multicenter Study for Induction of a Basic Care Bundle. Neurol Med Chir (Tokyo) 2017, 57:542-547.

3. Cho JS, Kim SH, Shin S, Pak HN, Yang SJ, Oh YJ: Effects of Dexmedetomidine on Changes in Heart Rate Variability and Hemodynamics During Tracheal Intubation. Am J Ther 2016, 23:e369-376.

4. Gorges M, Poznikoff AK, West NC, Brodie SM, Brant RF, Whyte SD: Effects of Dexmedetomidine on Blood Glucose and Serum Potassium Levels in Children Undergoing General Anesthesia: A Secondary Analysis of Safety Endpoints During a Randomized Controlled Trial. Anesth Analg 2019, 129:1093-1099.

5. Gorska-Warsewicz H, Rejman K, Laskowski W, Kowalcze K: Food Sources of Potassium in the Average Polish Diet. Nutrients 2019, 11.

6. Aantaa R, Scheinin M: Alpha 2-adrenergic agents in anaesthesia. Acta Anaesthesiol Scand 1993, 37:433448.

7. Chrysostomou C, Schulman SR, Herrera Castellanos M, Cofer BE, Mitra S, da Rocha MG, Wisemandle WA, Gramlich L: A phase II/III, multicenter, safety, efficacy, and pharmacokinetic study of dexmedetomidine in preterm and term neonates. $J$ Pediatr 2014, 164:276-282 e271-273.

8. Michaeli B, Martinez A, Revelly JP, Cayeux MC, Chiolero RL, Tappy L, Berger MM: Effects of endotoxin on lactate metabolism in humans. Crit Care 2012, 16:R139.

9. Peng Y, Zhu H, Chen H, Zhu Z, Zhou H, Zhang S, Gao L, Shi L, Li X, Luo Z: Dexmedetomidine attenuates acute paroxysmal sympathetic hyperactivity. Oncotarget 2017, 8:69012-69019.

10. Miyamoto K, Nakashima T, Shima N, Kato S, Ueda K, Kawazoe Y, Ohta Y, Morimoto T, Yamamura H, Investigators DT: Effect of Dexmedetomidine on Lactate Clearance in Patients With Septic Shock: A Subanalysis of a Multicenter Randomized Controlled Trial. Shock 2018, 50:162-166.

11. Zhao J, Zhou C: The protective and hemodynamic effects of dexmedetomidine on hypertensive cerebral hemorrhage patients in the perioperative period. Exp Ther Med 2016, 12:2903-2908.

12. Su F, Hammer GB: Dexmedetomidine: pediatric pharmacology, clinical uses and safety. Expert Opin Drug Saf 2011, 10:55-66.

13. Zheng D, Zhao S, Zhang N, Shi J: Brain protective effect and hemodynamics of dexmedetomidine hydrochloride in patients with intracranial aneurysm. Saudi J Biol Sci 2020, 27:1850-1855.

14. Oshima H, Nakamura M, Watanabe O, Yamamura T, Funasaka K, Ohno E, Kawashima H, Miyahara R, Goto $\mathrm{H}$, Hirooka Y: Dexmedetomidine provides less body motion and respiratory depression during sedation in double-balloon enteroscopy than midazolam. SAGE Open Med 2017, 5:2050312117729920.

15. Shukla U, Prabhakar T, Malhotra K, Srivastava D, Malhotra K: Intraperitoneal bupivacaine alone or with dexmedetomidine or tramadol for post-operative analgesia following laparoscopic cholecystectomy: A comparative evaluation. Indian J Anaesth 2015, 59:234-239.

16. Praveena BL, Bharathi B, Sahana VR: Intraperitoneal Ropivacaine with Dexmedetomidine or Fentanyl for Postoperative Analgesia Following Laparoscopic Cholecystectomy: A Comparative Randomized Trial. Anesth Essays Res 2019, 13:169-173.

17. Chen Z, Chen T, Ye H, Chen J, Lu B: Intraoperative dexmedetomidine-induced polyuria from a loading dose: a case report. J Int Med Res 2020, 48:300060520910643. 
18. Selvaraj S, Panneerselvam S: Unusual adverse effect of dexmedetomidine and its management. Indian J Anaesth 2018, 62:317-318.

19. Akashi N, Murahata Y, Hosokawa M, Hikasa Y, Okamoto Y, Imagawa T: Cardiovascular and renal effects of constant rate infusions of remifentanil, dexmedetomidine and their combination in dogs anesthetized with sevoflurane. J Vet Med Sci 2021, 83:285-296.

20. Jiang $Y$, Xia M, Huang Q, Ding D, Li Y, Zhang Z, Zhang X: Protective effect of dexmedetomidine against organ dysfunction in a two-hit model of hemorrhage/resuscitation and endotoxemia in rats. Braz $\mathrm{J} \mathrm{Med}$ Biol Res 2019, 52:e7905.

\section{Tables}

Table 1 Patient demographic data

\begin{tabular}{|c|c|c|c|c|c|}
\hline & Group $D_{1}$ & Group $D_{2}$ & Group $D_{3}$ & Group C & $P$ \\
\hline Age (years) & $66.4 \pm 8.6$ & $64.6 \pm 9.2$ & $66.8 \pm 10.1$ & $67.5 \pm 8.5$ & 0.822 \\
\hline Gender (Male/Female) & $14 / 17$ & $14 / 16$ & $16 / 15$ & $12 / 19$ & 0.566 \\
\hline Height (cm) & $163.4 \pm 5.4$ & $166.9 \pm 6.5$ & $163.6 \pm 7.0$ & $167.5 \pm 6.8$ & 0.147 \\
\hline Weight (kg) & $62.9 \pm 8.2$ & $58.5 \pm 18.0$ & $62.3 \pm 5.5$ & $62.6 \pm 8.4$ & 0.958 \\
\hline $\mathrm{BMI}\left(\mathrm{kg} / \mathrm{m}^{2}\right)$ & $23.5 \pm 2.5$ & $21.9 \pm 3.5$ & $23.4 \pm 2.9$ & $22.3 \pm 2.3$ & 0.248 \\
\hline 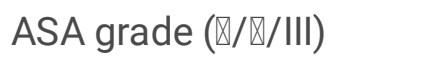 & $5 / 18 / 8$ & $9 / 18 / 3$ & $7 / 17 / 7$ & $7 / 20 / 4$ & 0.611 \\
\hline
\end{tabular}

Data are expressed as mean \pm SD or number. BMI: body mass index. ASA: American Society of Anesthesiologists.

Table 2 Patient intraoperative data 


\begin{tabular}{|llllll|}
\hline & Group $\mathrm{D}_{1}$ & Group $\mathrm{D}_{2}$ & Group $\mathrm{D}_{3}$ & Group C & $P$ \\
\hline Type of surgery $(\mathrm{A} / \mathrm{B})$ & $13 / 18$ & $12 / 18$ & $10 / 21$ & $11 / 20$ & 0.661 \\
\hline Mode of operation $\left(\mathrm{A}^{\prime} / \mathrm{B}^{\prime}\right)$ & $10 / 21$ & $9 / 21$ & $10 / 21$ & $8 / 23$ & 0.721 \\
\hline Operation time $(\mathrm{min})$ & $223.2 \pm 41.9$ & $227.6 \pm 41.9$ & $231.3 \pm 61.5$ & $225.8 \pm 37.6$ & 0.980 \\
& & & & & \\
\hline Anesthesia time $(\mathrm{min})$ & $282.8 \pm 79.9$ & $291.8 \pm 73.0$ & $291.6 \pm 39.7$ & $286.9 \pm 36.9$ & 0.952 \\
\hline Extubation time $(\mathrm{min})$ & $14.7 \pm 7.6$ & $14.4 \pm 10.1$ & $12.2 \pm 6.2$ & $9.9 \pm 3.9$ & 0.251 \\
\hline Total liquid volume $(\mathrm{mL})$ & $2300.0 \pm 313.8$ & $2323.5 \pm 393.0$ & $2362.5 \pm 453.7$ & $2263.2 \pm 305.9$ & 0.155 \\
\hline Urine volume $(\mathrm{mL})$ & $642.9 \pm 361.0$ & $797.1 \pm 376.0$ & $706.3 \pm 321.4$ & $476.3 \pm 225.1$ & 0.030 \\
\hline Bleeding volume $(\mathrm{mL})$ & $101.4 \pm 68.7$ & $116.5 \pm 68.8$ & $176.9 \pm 179.1$ & $110.5 \pm 83.7$ & 0.210 \\
\hline
\end{tabular}

Data are expressed as mean \pm SD or number. A: Radical resection for gastric tumor, B: Radical resection of colorectal tumor, A': Laparotomy, B': Laparoscopic surgery.

Table 3 Changes of potassium level and Lactate level in the four groups at different time points 


\begin{tabular}{|c|c|c|c|c|c|c|c|c|c|}
\hline & & Group $D_{1}$ & Group $D_{2}$ & Group $D_{3}$ & Group C & $P_{a}$ & $P_{b}$ & $P_{c}$ & $P_{d}$ \\
\hline \multirow{9}{*}{$\begin{array}{l}\text { Potassium } \\
\text { level }\end{array}$} & $\mathrm{T}_{1}$ & $3.67 \pm 0.30$ & $3.65 \pm 0.40$ & $3.71 \pm 0.31$ & $3.79 \pm 0.35$ & 0.338 & 0.243 & 0.516 & 0.639 \\
\hline & $T_{6}$ & $3.71 \pm 0.39$ & $3.70 \pm 0.36$ & $3.87 \pm 0.43$ & $3.82 \pm 0.43$ & 0.700 & 0.294 & 0.911 & 0.226 \\
\hline & $\mathrm{T}_{7}$ & $3.75 \pm 0.46$ & $3.67 \pm 0.44$ & $4.04 \pm 0.53$ & $3.89 \pm 0.50$ & 0.421 & 0.169 & 0.386 & 0.036 \\
\hline & $\mathrm{T}_{10}$ & $3.84 \pm 0.27$ & $3.71 \pm 0.20$ & $3.96 \pm 0.48$ & $3.80 \pm 0.28$ & 0.977 & 0.116 & 0.897 & 0.056 \\
\hline & $\mathrm{T}_{11}$ & $4.07 \pm 0.26$ & $4.03 \pm 0.25$ & $4.21 \pm 0.41$ & $4.25 \pm 0.30$ & 0.088 & 0.032 & 0.629 & 0.109 \\
\hline & $\begin{array}{l}P_{\mathrm{T} 1-} \\
6\end{array}$ & 0.620 & 0.601 & 0.056 & 0.431 & & & & \\
\hline & $\begin{array}{l}P_{\mathrm{T} 1-} \\
7\end{array}$ & 0.470 & 0.902 & 0.008 & 0.180 & & & & \\
\hline & $\begin{array}{l}P_{\mathrm{T} 1-} \\
10\end{array}$ & 0.077 & 0.225 & 0.015 & 0.854 & & & & \\
\hline & $\begin{array}{l}P_{\mathrm{T} 1-} \\
11\end{array}$ & 0.003 & 0.002 & $\llbracket 0.001$ & $\llbracket 0.001$ & & & & \\
\hline \multirow{7}{*}{$\begin{array}{l}\text { Lactate } \\
\text { level }\end{array}$} & $\mathrm{T}_{1}$ & $0.84 \pm 0.30$ & $1.06 \pm 0.31$ & $0.86 \pm 0.26$ & $0.82 \pm 0.44$ & 0.497 & 0.246 & 0.600 & 0.052 \\
\hline & $T_{6}$ & $0.74 \pm 0.29$ & $0.76 \pm 0.36$ & $0.71 \pm 0.28$ & $0.78 \pm 0.35$ & 0.860 & 0.901 & 0.785 & 0.693 \\
\hline & $\mathrm{T}_{7}$ & $0.78 \pm 0.42$ & $1.01 \pm 0.45$ & $0.83 \pm 0.32$ & $0.80 \pm 0.29$ & 0.949 & 0.055 & 0.724 & 0.136 \\
\hline & $\mathrm{T}_{10}$ & $1.02 \pm 0.61$ & $1.13 \pm 0.53$ & $0.81 \pm 0.27$ & $0.94 \pm 0.37$ & 0.639 & 0.493 & 0.176 & 0.033 \\
\hline & $\begin{array}{l}P_{\mathrm{T} 1-} \\
6\end{array}$ & 0.248 & 0.002 & 0.003 & 0.133 & & & & \\
\hline & $\begin{array}{l}P_{\mathrm{T} 1-} \\
7\end{array}$ & 0.251 & 0.799 & 0.599 & 0.559 & & & & \\
\hline & $\begin{array}{l}P_{\mathrm{T} 1-} \\
10\end{array}$ & 0.739 & 0.579 & 0.338 & 0.153 & & & & \\
\hline
\end{tabular}

Data are expressed as mean $\pm \mathrm{SD} . P_{\mathrm{a}}, P_{\mathrm{b}}$ and $P_{\mathrm{c}}$ for comparison between group $\mathrm{C}$ and groups $\mathrm{D}_{1}, \mathrm{D}_{2}$ and $\mathrm{D}_{3}$, respectively. $P_{\mathrm{d}}$ for comparison between Groups $\mathrm{D}_{2}$ and $\mathrm{D}_{3}$.

Table 4 Perioperative changes of heart rate 


\begin{tabular}{|c|c|c|c|c|c|c|c|c|}
\hline & Group $D_{1}$ & Group $D_{2}$ & Group $D_{3}$ & Group C & $P_{\mathrm{a}}$ & $P_{\mathrm{b}}$ & $P_{\mathrm{c}}$ & $P_{\mathrm{d}}$ \\
\hline $\mathrm{T}_{1}$ & $73.0 \pm 11.6$ & $72.4 \pm 10.5$ & $72.9 \pm 7.8$ & $70.5 \pm 13.0$ & 0.515 & 0.609 & 0.510 & 0.229 \\
\hline $\mathrm{T}_{2}$ & $65.5 \pm 8.8$ & $69.2 \pm 16.3$ & $67.3 \pm 8.8$ & $71.1 \pm 14.3$ & 0.217 & 0.652 & 0.377 & 0.679 \\
\hline $\mathrm{T}_{3}$ & $61.7 \pm 10.0$ & $59.6 \pm 9.7$ & $59.4 \pm 11.2$ & $65.8 \pm 14.2$ & 0.321 & 0.113 & 0.110 & 0.967 \\
\hline $\mathrm{T}_{4}$ & $61.9 \pm 9.9$ & $58.8 \pm 9.8$ & $61.0 \pm 7.3$ & $63.8 \pm 12.8$ & 0.599 & 0.149 & 0.419 & 0.477 \\
\hline $\mathrm{T}_{5}$ & $62.4 \pm 9.0$ & $57.9 \pm 8.0$ & $59.9 \pm 8.4$ & $67.6 \pm 15.5$ & 0.187 & 0.010 & 0.042 & 0.490 \\
\hline$T_{6}$ & $61.3 \pm 7.5$ & $59.8 \pm 5.3$ & $58.9 \pm 7.8$ & $64.0 \pm 20.5$ & 0.540 & 0.314 & 0.231 & 0.702 \\
\hline $\mathrm{T}_{7}$ & $66.4 \pm 8.9$ & $62.2 \pm 7.4$ & $59.8 \pm 7.3$ & $69.8 \pm 12.4$ & 0.305 & 0.017 & 0.002 & 0.350 \\
\hline $\mathrm{T}_{8}$ & $70.1 \pm 8.6$ & $66.4 \pm 9.4$ & $66.6 \pm 10.4$ & $76.1 \pm 11.1$ & 0.094 & 0.005 & 0.007 & 0.965 \\
\hline $\mathrm{T}_{9}$ & $70.9 \pm 10.3$ & $67.2 \pm 10.3$ & $67.3 \pm 10.5$ & $75.2 \pm 11.4$ & 0.257 & 0.030 & 0.034 & 0.983 \\
\hline $\mathrm{T}_{10}$ & $71.4 \pm 8.5$ & $62.6 \pm 8.9$ & $63.2 \pm 9.9$ & $71.6 \pm 12.6$ & 0.904 & 0.011 & 0.019 & 0.856 \\
\hline$P_{\mathrm{T} 1-2}$ & 0.002 & 0.321 & 0.025 & 0.799 & & & & \\
\hline$P_{\mathrm{T} 1-3}$ & 0.001 & $凶 0.001$ & 0.001 & 0.091 & & & & \\
\hline$P_{\mathrm{T} 1-4}$ & $凶 0.001$ & $凶 0.001$ & $凶 0.001$ & 0.023 & & & & \\
\hline$P_{\mathrm{T} 1-5}$ & $凶 0.001$ & $凶 0.001$ & $凶 0.001$ & 0.416 & & & & \\
\hline$P_{\mathrm{T} 1-6}$ & 0.003 & $凶 0.001$ & $\triangle 0.001$ & 0.177 & & & & \\
\hline$P_{\mathrm{T} 1-7}$ & 0.033 & $\llbracket 0.001$ & $\llbracket 0.001$ & 0.876 & & & & \\
\hline$P_{\mathrm{T} 1-8}$ & 0.484 & 0.030 & 0.024 & 0.148 & & & & \\
\hline$P_{\mathrm{T} 1-9}$ & 0.603 & 0.037 & 0.022 & 0.143 & & & & \\
\hline$P_{\mathrm{T} 1-10}$ & 0.545 & 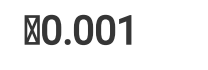 & 『0.001 & 0.650 & & & & \\
\hline
\end{tabular}

Data are expressed as mean $\pm \mathrm{SD} . P_{\mathrm{a}}, P_{\mathrm{b}}$ and $P_{\mathrm{c}}$ for comparison between group $\mathrm{C}$ and groups $\mathrm{D}_{1}, \mathrm{D}_{2}$ and $\mathrm{D}_{3}$, respectively. $P_{\mathrm{d}}$ for comparison between Groups $\mathrm{D}_{2}$ and $\mathrm{D}_{3}$.

Table 5 Perioperative changes of mean arterial pressure 


\begin{tabular}{|c|c|c|c|c|c|c|c|c|}
\hline & Group $D_{1}$ & Group $D_{2}$ & Group $D_{3}$ & Group C & $P_{\mathrm{a}}$ & $P_{\mathrm{b}}$ & $P_{\mathrm{c}}$ & $P_{\mathrm{d}}$ \\
\hline $\mathrm{T}_{1}$ & $104.7 \pm 14.8$ & $98.4 \pm 12.1$ & $100.3 \pm 10.4$ & $100.5 \pm 14.1$ & 0.465 & 0.625 & 0.961 & 0.633 \\
\hline $\mathrm{T}_{2}$ & $103.5 \pm 14.2$ & $97.1 \pm 11.5$ & $97.3 \pm 11.1$ & $95.2 \pm 13.4$ & 0.040 & 0.641 & 0.614 & 0.965 \\
\hline $\mathrm{T}_{3}$ & $97.9 \pm 15.2$ & $96.5 \pm 17.0$ & $92.8 \pm 11.7$ & $90.8 \pm 15.0$ & 0.162 & 0.253 & 0.697 & 0.472 \\
\hline $\mathrm{T}_{4}$ & $89.5 \pm 12.3$ & $97.4 \pm 16.1$ & $92.3 \pm 11.4$ & $98.6 \pm 15.4$ & 0.083 & 0.804 & 0.781 & 0.974 \\
\hline $\mathrm{T}_{5}$ & $91.9 \pm 11.8$ & $99.3 \pm 11.3$ & $97.8 \pm 15.0$ & $102.1 \pm 13.8$ & 0.045 & 0.530 & 0.343 & 0.750 \\
\hline $\mathrm{T}_{6}$ & $87.0 \pm 14.1$ & $92.1 \pm 13.4$ & $88.9 \pm 10.4$ & $88.2 \pm 14.8$ & 0.913 & 0.375 & 0.874 & 0.445 \\
\hline $\mathrm{T}_{7}$ & $86.1 \pm 17.3$ & $92.4 \pm 14.8$ & $92.4 \pm 13.8$ & $99.8 \pm 16.7$ & 0.018 & 0.157 & 0.169 & 0.987 \\
\hline $\mathrm{T}_{8}$ & $95.4 \pm 12.5$ & $96.2 \pm 15.9$ & $100.9 \pm 11.3$ & $111.9 \pm 17.7$ & 0.001 & 0.002 & 0.033 & 0.338 \\
\hline $\mathrm{T}_{9}$ & $94.7 \pm 11.7$ & $96.1 \pm 16.5$ & $100.6 \pm 13.1$ & $106.8 \pm 20.3$ & 0.030 & 0.049 & 0.258 & 0.386 \\
\hline $\mathrm{T}_{10}$ & $97.3 \pm 11.8$ & $90.8 \pm 14.0$ & $88.8 \pm 10.2$ & $99.5 \pm 12.4$ & 0.448 & 0.037 & 0.013 & 0.437 \\
\hline$P_{\mathrm{T} 1-2}$ & 0.678 & 0.553 & 0.136 & 0.236 & & & & \\
\hline$P_{\mathrm{T} 1-3}$ & 0.051 & 0.664 & 0.054 & 0.007 & & & & \\
\hline$P_{\mathrm{T} 1-4}$ & 0.002 & 0.817 & 0.351 & 0.585 & & & & \\
\hline$P_{\mathrm{T} 1-5}$ & 0.007 & 0.799 & 0.558 & 0.706 & & & & \\
\hline$P_{\mathrm{T} 1-6}$ & $凶 0.001$ & 0.125 & 0.006 & 0.002 & & & & \\
\hline$P_{\mathrm{T} 1-7}$ & $\llbracket 0.001$ & 0.139 & 0.145 & 0.880 & & & & \\
\hline$P_{\mathrm{T} 1-8}$ & 0.047 & 0.550 & 0.867 & 0.015 & & & & \\
\hline$P_{\mathrm{T} 1-9}$ & 0.048 & 0.543 & 0.951 & 0.215 & & & & \\
\hline$P_{\mathrm{T} 1-10}$ & 0.132 & 0.029 & 0.002 & 0.750 & & & & \\
\hline
\end{tabular}

Data are expressed as mean $\pm \mathrm{SD} . P_{\mathrm{a}}, P_{\mathrm{b}}$ and $P_{\mathrm{c}}$ for comparison between group $\mathrm{C}$ and groups $\mathrm{D}_{1}, \mathrm{D}_{2}$ and $\mathrm{D}_{3}$, respectively. $P_{\mathrm{d}}$ for comparison between Groups $\mathrm{D}_{2}$ and $\mathrm{D}_{3}$.

Table 6 Changes of liver and kidney function in four groups 


\begin{tabular}{|c|c|c|c|c|c|}
\hline & & Group $D_{1}$ & Group $D_{2}$ & Group $D_{3}$ & Group C \\
\hline \multirow{3}{*}{$\begin{array}{l}\text { ALT } \\
(\mathrm{U} / \mathrm{L})\end{array}$} & $\mathrm{T}_{1}$ & $15.4 \pm 7.7$ & $17.7 \pm 9.9$ & $22.8 \pm 22.5$ & $15.3 \pm 8.2$ \\
\hline & $\mathrm{T}_{11}$ & $44.0 \pm 42.7$ & $28.7 \pm 8.6$ & $48.1 \pm 40.0$ & $49.3 \pm 51.3$ \\
\hline & $P$ & 0.030 & $\llbracket 0.001$ & 0.005 & 0.011 \\
\hline \multirow{3}{*}{$\begin{array}{l}\text { AST } \\
(\mathrm{U} / \mathrm{L})\end{array}$} & $\mathrm{T}_{1}$ & $18.8 \pm 3.3$ & $21.3 \pm 6.5$ & $24.3 \pm 15.2$ & $18.5 \pm 5.84$ \\
\hline & $\mathrm{T}_{11}$ & $45.1 \pm 52.7$ & $25.4 \pm 8.7$ & $46.1 \pm 35.6$ & $47.4 \pm 51.3$ \\
\hline & $P$ & 0.081 & 0.037 & 0.034 & 0.031 \\
\hline \multirow[t]{3}{*}{ Serum creatinine $(\mu \mathrm{mol} / \mathrm{L})$} & $\mathrm{T}_{1}$ & $64.0 \pm 11.3$ & $64.1 \pm 10.6$ & $67.5 \pm 15.3$ & $70.5 \pm 15.1$ \\
\hline & $\mathrm{T}_{11}$ & $58.4 \pm 10.8$ & $65.3 \pm 9.4$ & $64.6 \pm 13.7$ & $69.7 \pm 13.1$ \\
\hline & $P$ & 0.052 & 0.565 & 0.479 & 0.069 \\
\hline \multirow[t]{3}{*}{ Blood urea nitrogen $(\mathrm{mmol} / \mathrm{L})$} & $\mathrm{T}_{1}$ & $5.9 \pm 1.6$ & $5.1 \pm 1.5$ & $5.5 \pm 3.4$ & $4.9 \pm 2.3$ \\
\hline & $\mathrm{T}_{11}$ & $4.8 \pm 1.6$ & $4.7 \pm 1.8$ & $4.9 \pm 1.3$ & $4.8 \pm 2.2$ \\
\hline & $P$ & 0.014 & 0.361 & 0.529 & 0.940 \\
\hline
\end{tabular}

Data are expressed as mean \pm SD. ALT: alanine aminotransferase. AST: aspartate aminotransferase. Figures 


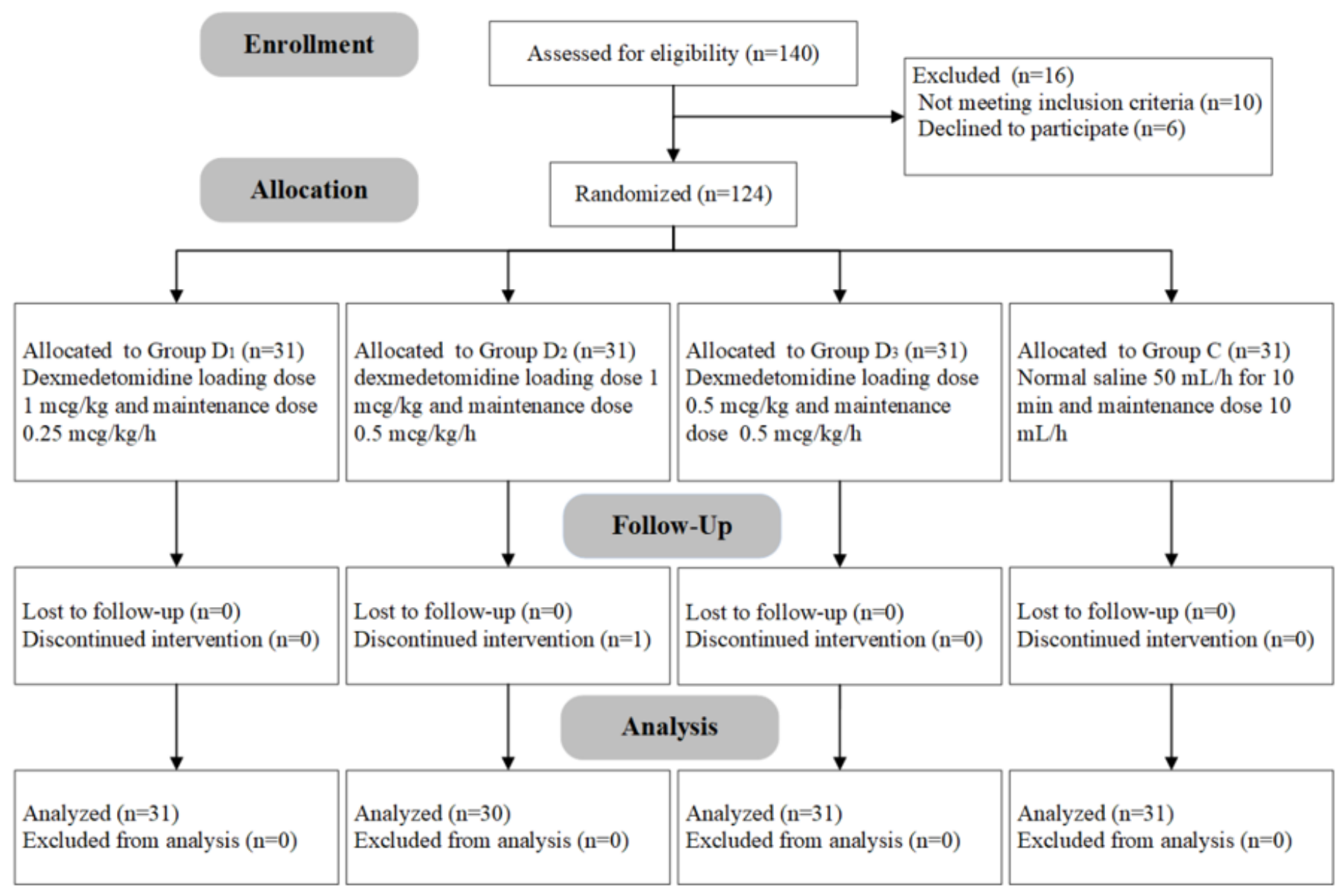

\section{Figure 1}

Consort flow diagram for the study. 